University Hospital and during 2010-2013 in other Finnish University Hospitals (Helsinki, K uopio, Tampere, Turku). DNA samples were genotyped using the Illumina HumanCoreExome BeadChip consisting of approximately 550,000 single-nucleotide polymorphisms (SNPs); after quality control, 60 cases (moderate-severe BPD) and 114 controls (no or mild BPD) remained for a genome-wide association study (GWAS). In the next step, approximately 200 SNPs showing suggestive signals are genotyped in additional infants $(n=116 / 232)$ to determine which associations are replicated.

Results In GWAS, we detected suggestive association signals ( $\mathrm{p}<$ $1^{-4}$ ) for several SNPs; many of these SNPs were located within or near genes that can be considered as plausible candidate genes for BPD (e.g. the CRP and PTPN6 genes encoding C-reactive protein and protein-tyrosine phosphatase SHP-1, respectively). Some of the SNPs showing suggestive associations in two previous GWASs of BPD showed weak associations (e.g. those within the PALM2 and CTNNA3 genes).

Conclusions In genome-wide association study of BPD, we detected several suggestive associations. These initial results require verification in subsequent studies, including replication in additional populations and functional studies of the arising candidate genes.

\section{PO-0745 CONGENITAL DIAPHRAGMATIC HERNIA IN NEONATES - FIRST TIME ASSESSMENT OF OUTCOME AMONG PALESTINIAN BABIES}

${ }^{1} \mathrm{H}$ Khamash, ${ }^{2} \mathrm{~A}$ Atawna, ${ }^{3} \mathrm{R}$ Al-Sharif, ${ }^{4} \mathrm{~S}$ Abdelrazeq, ${ }^{5} \mathrm{~N}$ Al Deesi. ${ }^{1}$ Pediatric, Al-Quds University, Jerusalem, Palestine (Via Israel); ${ }^{2}$ Neonatology, Makassed Hospital, Jerusalem, Palestine (Via Israel); ${ }^{3}$ School of Medicine, Al-Quds University, Jerusalem, Palestine (Via Israel); ${ }^{4}$ Pediatrics, Makassed Hospital, Jerusalem, Palestine (Via Israel); ${ }^{5}$ Pediatric Surgery, Makassed Hospital, Jerusalem, Palestine (Via Israel)

10.1136/archdischild-2014-307384.1384

Objectives To review our NICU and surgical experience of all cases of $\mathrm{CDH}$ that was admitted to our hospital in last 10 years. To identify the risk factors for morbidity and mortality.

Setup Makassed Charitable Hospital, main tertiary hospital for Palestinian Territories (West Bank, Gaza and East Jerusalem).

Material and methods Retrospective Review of all cases of CDH admitted to our NICU between January 2003 and December 2013. Data about: Antenatal diagnosis, gestational age, birth weight, Apgar score, Blood gas at delivery, time of surgery, finding at surgery, ventilation days, mortality outcome and several other items were recorded and analysed. Primary outcome was: mortality, total days of ventilation and total days of Oxygen requirement.

Results Thirty three $(\mathrm{n}=33)$ cases of $\mathrm{CDH}$ were reviewed. Twenty two cases were inborn (67\%). Mortality was 10 cases (30\%). Twenty three cases were discharged home alive and off oxygen therapy (70\%). Better survival rate after 2009 (77\% Vs $63 \%$ ) this could be due to gentle ventilation strategies, better use of antenatal ultrasound for diagnosis. Lower mortality and less ventilation days were significantly associated with lower initial PaCO2 $(p<0.001)$ and higher PH $(p<0.001)$ and higher Gestational age (P 0.05). Presence of liver and/or spleen as part of hernia contents correlates negatively with the primary outcome $(\mathrm{p}<0.05)$.

Conclusion This is the first study that outlines the mortality and morbidity and their risk factors in Palestinian Territories with no ECMO is used demonstrating fairly good outcome with gentle ventilation strategies and antenatal diagnosis.

\section{PO-0746 RESPIRATORY SUPPORT IN TERM NEWBORNS AFTER C-SECTION}

D Konstantelos, J Dinger, S Ifflaender, M Rüdiger. Department of Neonatology and Pediatric Intensive Care, Medizinische Fakultät Carl Gustav Carus an Der TU Dresden, Dresden, Germany

\subsection{6/archdischild-2014-307384.1385}

Background and aims After c-section term newborns are at risk of respiratory problems. Whereas some newborns require respiratory support only for a short time in the delivery room (DR), others are admitted to the NICU for prolonged therapy. Our aim was to compare differences between newborns with respiratory support in DR only and those admitted to the NICU.

Methods Retrospective analysis of video recorded DR-management of term newborns born between January 2012 and November 2013 via c-section.

Results 368 newborns were analysed with 82 (22\%) receiving respiratory support. From them, $26(32 \%)$ were transported to NICU for further treatment, the remaining 56 (68\%) were stabilised after a short period of CPAP treatment. There were no demographic differences between both groups. CPAP-administration started after a median of $3.4(0.2-27)$ in NICU and 3.7 (0.03-17) minutes in DR infants. At the start of CPAP administration infants had a median heart rate of 161 (75-195) in NICU and 153 (56-200) in DR newborns and SpO2 of 69 (41$100)$ and $80(55-100)$ respectively $(\mathrm{p}=0.01) .8(31 \%)$ NICU and 15 (27\%) DR newborns received a sustained inflation; mechanical ventilation via face-mask received 4 and 6 newborns respectively. In infants remaining in the DR respiratory support was stopped after a median of $7.6(0.2-21)$ minutes, infants were transferred to the NICU after a respiratory support of 17.7 (4-29.6) minutes respectively.

Discussion Except for lower $\mathrm{SpO}_{2}$ values there are no parameters to predict the need for the length of treatment in respiratory depressed term newborns.

\section{PO-0747 THE STUDY OF OXIDATIVE STRESS AT PRETERM NEWBORNS WITH RESPIRATORY DISTRESS SYNDROME}

M Matyas, L Blaga, M Hasmasanu, G Zaharie. Neonatology Department, University of Medicine and Pharmacy, CLUJ-NAPOCA, Romania

\subsection{6/archdischild-2014-307384.1386}

Aim The diseases of newborns which involve oxidative stress are: respiratory distress (RDS), bronchopulmonary dysplasia, retinopathy and necrotizing enterocolitis. The aim of the study was to evaluate the oxidative stress trough the lipid peroxidation at preterm newborns with RDS.

Material and methods We conducted a prospective, non -randomised study. The study group was represented by sixty preterm newborns with RDS. The control was represented by 20 healthy late preterm newborns. For all patients family's consent was obtained. The study of the oxidative stress was performed by the measurement of malonildyaldehide (MDA) by Satoh's method. For each newborn we determined the MDA on the first and third day of life. For the control was carried out one determination on the first day of life. The statistical analysis was done using the SPSS program.

Results The RDS was present in mild form at 35\% newborns, medium form at $42 \%$ and severe form at $23 \%$. Seven newborns presented neonatal septicemia. Cerebral haemorrhage was present at 12 newborns of the study group. At 13 preterm the 